\title{
The prediction of moisture through the use of neural networks MLP type
}

\author{
Hicham El Badaoui ${ }^{1}$, Abdelaziz Abdallaoui ${ }^{1 *}, \operatorname{Imad}$ Manssouri ${ }^{2}$, \\ Habiba Ousmana ${ }^{1}$ \\ ${ }^{1}$ Laboratory of Chemical Biology Applied to the Environment, Analytical Chemistry and Environment Team, \\ Faculty of Sciences, University Moulay Ismail, BP 11201, Zitoune, Meknes -Morocco. \\ ${ }^{2}$ Mechanical Engineering Department and Structures, ENSAM, University Moulay Ismail, BP 4042, 50000, \\ Meknes, Morocco.
}

\begin{abstract}
Neural networks are powerful techniques for non-linear data, which have been proven in many domains. As a result, the artificial neural networks have been applied in various fields including meteorology and climatology. This work is a contribution to the development of methods of weather prediction in general, and humidity rate in particular. In a first step, methods that are based on the study of artificial neural networks types MLP (Multilayer Perceptron) are applied for the prediction of moisture in the area of Chefchaouen in Morocco. In a second step, the proposed new architecture of neural networks of MLP type was compared to the model of multiple linear regression (MLR). The basis of learning neural model was collected between 2008 and 2011 during 1248 days. The latter consists of a number of climatic parameters, such as atmospheric pressure, air temperature, visibility, cloud cover, precipitation, dew point temperature, wind speed and humidity.Predictive models established by the MLP neural networks method are more powerful compared to those established by multiple linear regression, because of the fact that good correlation was obtained with the parameters from a neural approach with a mean squared error $5 \%$.
\end{abstract}

Keywords: Humidity, Artificial Neural Networks, MLP, Linear regression, Prediction.

\section{INTRODUCTION}

The moisture content is the amount of water vapor that is in the air. In meteorology, it is important to know this parameter, among others, as it provides information on the possible formation of clouds and precipitation.

In the field of meteorology, the problems of prediction are common. However, the relation between the variables is rarely linear. The interaction between the parameters, even if it is suspected, is difficult to model. The uses of artificial neural networks present an interesting alternative to traditional statistics. Indeed, with these conventional methods, it is necessary to think of a model and test it, think of another model until we obtain a sufficiently accurate model.

In the literature, several prediction methods are proposed for the prediction of meteorological parameters. We mention as examples some items proposed in the field of prediction using neural networks type multilayer Perceptron (MLP) networks and Radial Basis Function (RBF).

Cheggaga et al. (2010), [1] showed the possibility of using neural networks MLP type layered nonrecurring the extrapolation, the prediction and the interpolation of wind speed in time and space in 3D (radius $\mathrm{r}$, height $\mathrm{h}$, time $\mathrm{t}$ ), based on a neural network learning a few days.

Nohair et al. (2008), [2] have used the neural networks for the prediction variations in the temperature of a stream as a function of climatic variables, such as temperature of the ambient air and the flow of water received by the current of water. Two methods were applied: the first type - iterative- and the second method consist of estimating the temperature of all days considered at once.

Voyant et al. (2011), [3] have applied the network type feed-forward to predict solar radiation and photovoltaic power generation from artificial neural networks. As input data, they used the rental month, the average pressure, and average temperature, the average value of the wind speed and the mean duration of sunshine.

Ryad et al. (2002), [4] have worked on the application of neural network RBF (Radial Basis Function) for the prediction problem of a nonlinear system. The results of their work have made a contribution at the recurrent network topology to accommodate the dynamic data and a contribution for the improvement of the learning algorithm.

Perez et al. (2001), [5] have proposed that the concentration of nitrite ion $\mathrm{NO}_{2}^{-}$and nitric oxide $\mathrm{NO}$ in Santiago based on meteorological variables and using the linear regression method and neural network method. The results showed that the neural network is the method that achieves the lowest prediction error compared to other methods. 
Bélanger et al. (2005), [6] have treated a comparative study of the performance of two modeling methods used to predict the temperature of water from hydrometeorological parameters based on the model of artificial intelligence and the traditional method of multiple linear regression. The results of this study showed that the artificial neural networks seem to give a slightly better fit to the data than offered by the multiple linear regression. In this work, we propose to develop a statistical model nonlinear prediction of moisture in the region of Chefchaouen in Morocco according to meteorological variables.

\section{MATERIALS AND METHODS}

\section{II.1 Presentation of the study zone}

The region of Chefchaouen, situated in the north-west of Morocco (Latitude: $35^{\circ} 10^{\prime} 17$ North, Longitude: $5^{\circ} 16^{\prime} 11$ West, Elevation: 564 Meters) of the Rif chain, is bounded on the north by the Mediterranean at a length of $120 \mathrm{~km}$, on the south by the provinces of Taounate and Sidi Kacem, on the east by the province of $\mathrm{Al}$ Hoceima and on the west by the provinces of Tetuan and Larache. It is a province that represents the administrative region of Tangier-Tetouan. It is located 600 Meters (Fig.1).

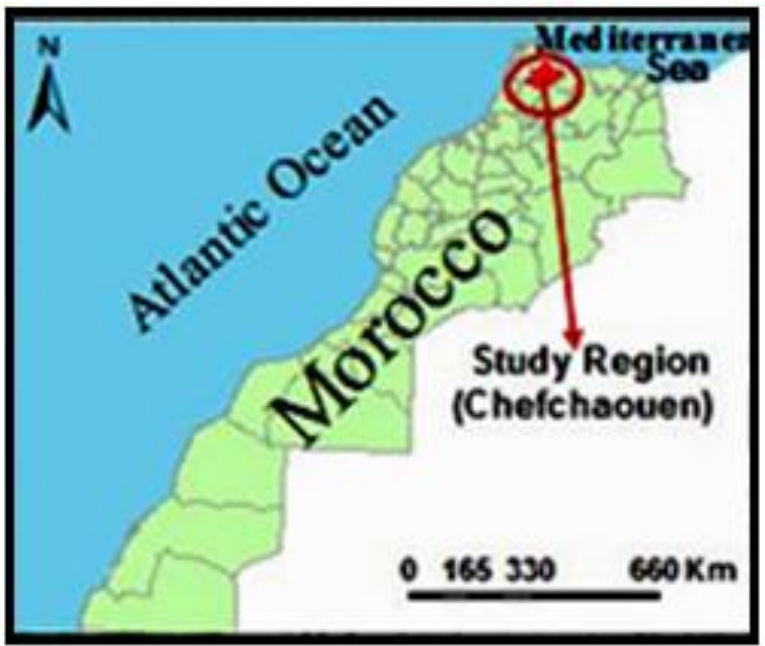

II.2 Data base

Fig.1. Geographic situation of the region of Chefchaouen. Chefchaouen:

The database used in this work consists of the values of eight meteorological variables for the region of

$\checkmark$ Seven independent variables (explanatory): the air temperature, dew point temperature, air pressure, visibility, cloud cover, wind speed and rain.

$\checkmark$ A dependent variable (to explain): humidity.

These values were taken every three hours for 1248 days during the years 2008, 2009, 2010 and 2011. They were converted to daily averages for all variables except for the amount of precipitation (rain) which was transformed into a cumulative value of the day. Fig. 2 shows the evolution of humidity during the period.

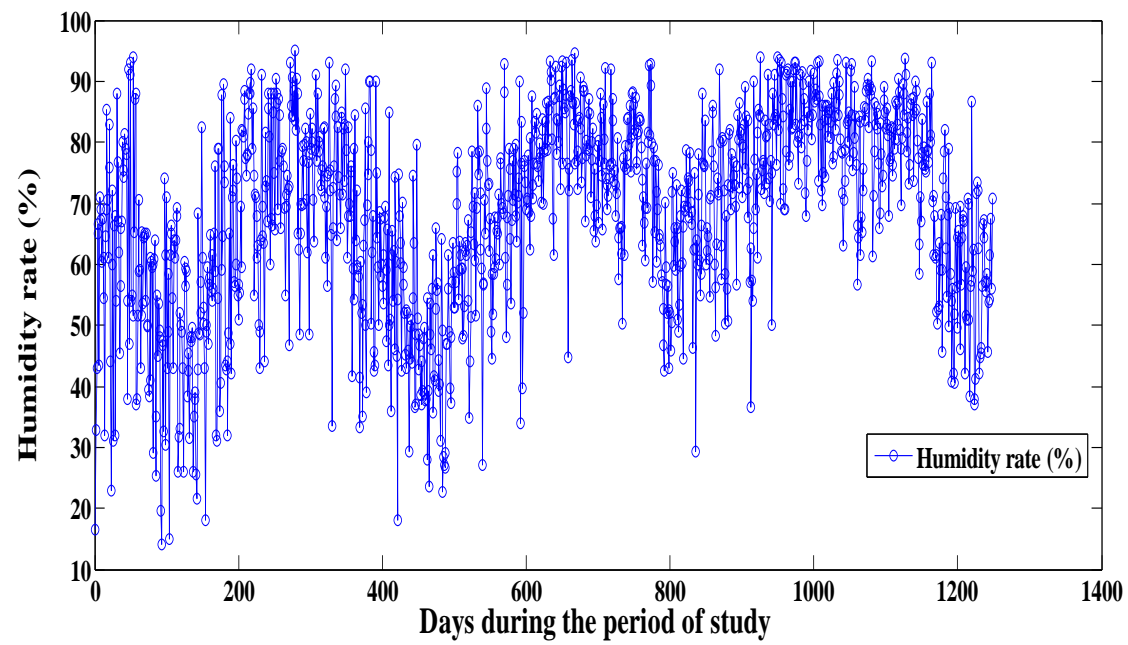

Fig.2. Variation of moisture during the study period. 


\section{II.3 Formatting data}

The input data (independent or explained variables) are unprocessed raw values. They have very different orders of magnitude depending on the variables. To standardize the measurement scales, these data are converted into standardized variables. Indeed, the values of each independent variable (i) were standardized relative to its mean and standard deviation according to the relation:

$$
X_{S}(i)=\frac{X(i)-X_{m}(i)}{\sigma_{X}(i)}
$$

With,

$\mathrm{X}_{\mathrm{S}}(\mathrm{i})$ : standardized value relating to the variable $\mathrm{i}$;

$\mathrm{X}(\mathrm{i})$ : Gross value relating to the variable $\mathrm{i}$;

$\mathrm{X}_{\mathrm{m}}(\mathrm{i})$ : Average;

$\sigma_{X}(i)$ : Standard deviation relating to the variable $\mathrm{i}$;

The standardization of values for all variables aims to avoid exponential calculations, very large or very small, and limits the increase in the average variance. To improve the performance of neural networks, it is better to normalize the values for the dependent variables so that they are in the interval $[0,1]$. This was done in order to adapt the dependent variables to the requirements of transfer function (sigmoid function). This normalization was performed according to the relation [7]:

$\mathrm{Y}_{\mathrm{n}}$ : Standard values,

$$
Y_{n}=\frac{\left(Y-Y_{\min }\right)}{\left(Y_{\max }-Y_{\min }\right)}
$$

$\mathrm{Y}$ : Original values,

$\mathrm{Y}_{\min }$ : Minimum value,

$\mathrm{Y}_{\max }$ : Maximum value.

\section{II.4 Artificial Neural Networks}

An artificial neural network is a computational model whose original inspiration was a biological model. Mathematical and computer representation of a biological neuron in the neural networks is called formal neuron; we have diagrammed the structure of an artificial neuron in Fig. 3.

We can then characterize a formal neuron by: A combination function, or an adder which performs the weighted $\operatorname{sum} A_{j}$. The weighted sum is equal to:

$$
A_{j}=\sum_{i=1}^{n} W_{i j} X_{i}
$$

$\mathrm{W}_{\mathrm{ij}}$ is the synaptic weight and $\mathrm{X}_{\mathrm{i}}$ is the input value on the variable $\mathrm{i}$. This is the sum of weighted activation that converges to the neuron $\mathrm{j}$; An activation function (or transfer) $\mathrm{f}$ which animates the neuron by determining its activation; An activation $Y_{j}$, equivalent to the output neuron. It is equal to:

$\theta_{\mathrm{j}}$ is the bias of neuron $\mathrm{j}$.

$$
Y_{j}=f\left(\sum_{i=1}^{n} W_{i j} X_{i}+\theta_{j}\right)
$$

There are several activation functions (hyperbolic tangent, Gaussian, sigmoid ...), but the most widely used is the sigmoid function [8], [9], [10] and [11]. It is written in the following form:

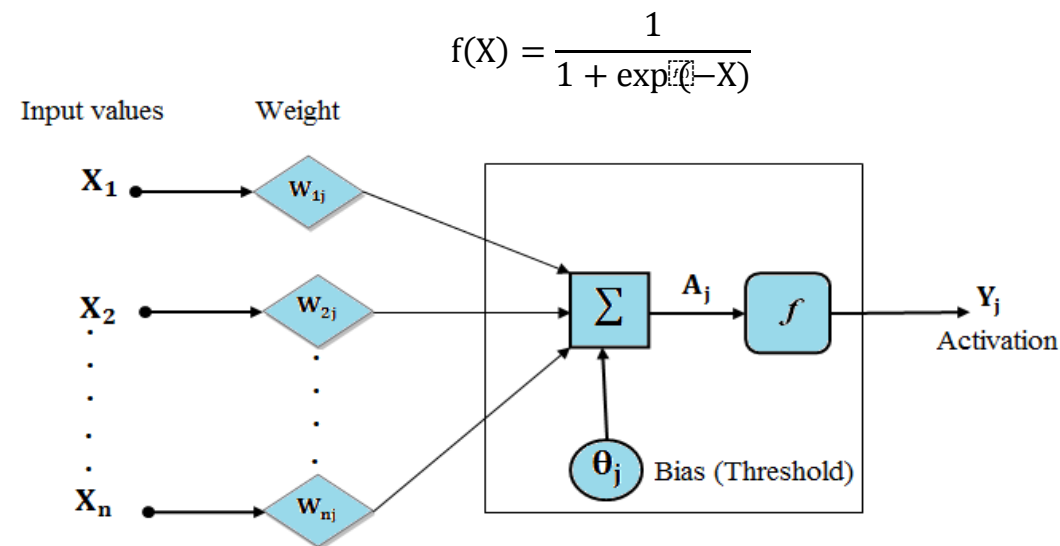

Fig.3. Structure of an artificial neuron. 


\section{II.5 Choice of the neural network}

Our choice is focused on a multi-layer non-recurring network. This one consists of three layers of neurons, called input layer, hidden layer and output layer. Fig. 4 shows the architecture of the network:

Many learning processes are available. In our case, we used the rule learning called learning by back propagation [6]. The purpose of this learning algorithm is to minimize the overall error E defined by:

$$
E=\frac{1}{2} \sum_{i, j=1}^{N}\left(Y_{j}-d_{i}\right)^{2}
$$

$\mathrm{Y}_{\mathrm{j}}$ : The output obtained by the network.

$\mathrm{d}_{\mathrm{i}}$ : The target (desired output).

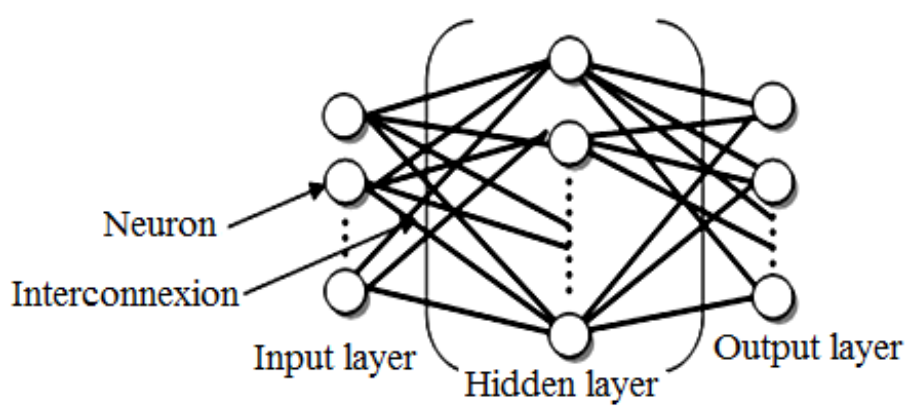

Fig.4. Simple diagram of a multilayer neural network.

The selected number of hidden neurons (NHN) in our network type determines the degree of learning. The correct number will be chosen by tests performed by the computer program. To create the optimal structure of the neural network, we changed the number of neurons in the hidden layer, playing on the parameters of our calculation program (number of iterations, learning coefficient, learning rate [12]. Next, we conducted tests to find the network structure which gives more efficient models with a network of three layers of Multilayer Perceptron type (MLP). in this layer.

An input layer formed by seven neurons representing the independent variables. No calculation is done

A hidden layer, where all calculations of parameter optimization of neural networks are made. In this layer, we changed the number of hidden neurons ( $\mathrm{NHN}=2,3,4,5,6,8,10,14 \ldots$. To 20), but during testing with $\mathrm{NHN}=4$, we obtained very effective results of calculation (see Table I).

An output layer of the network which represents the moisture content. The calculation result and the performance of established models are recorded in the output layer.

Table I: Changes in learning rate ( $\eta$ ), the number of iteration, the mean square error (MSE) for four neurons in the hidden layer.

\begin{tabular}{|c|c|c|c|c|}
\hline \multicolumn{5}{|c|}{ NHN = 4 } \\
\hline Learning rate $(\eta)$ & $\begin{array}{c}\text { Number of } \\
\text { iterations }\end{array}$ & $\begin{array}{c}\text { MSE } \\
\text { (learning error })\end{array}$ & $\begin{array}{c}\text { MSE validation } \\
\text { error }\end{array}$ & $\begin{array}{c}\text { MSE test } \\
\text { error }\end{array}$ \\
\hline $\mathbf{0 . 0 0 0 1}$ & $\mathbf{1 5}$ & $\mathbf{0 . 0 0 1 9 7 6}$ & $\mathbf{0 . 0 0 1 6 3 0}$ & $\mathbf{0 . 0 0 1 6 8 8}$ \\
\hline 0.1 & 18 & 0.135985 & 0.076888 & 0.268149 \\
\hline 0.1 & 30 & 0.170279 & 0.259533 & 0.168729 \\
\hline
\end{tabular}

For this study, we used a feed forward network with two layers, with a sigmoid transfer function in the hidden layer and a linear transfer function in the output layer. Previous work has shown that this pair of functions can approximate virtually all types of non-linear relationships [13]. Among the various network configurations tested, we selected one producing the lowest mean square error (MSE noted, Mean square error) of the entire database. The results that are listed in Table I are found after several iterations. Figure 5 shows the variation of mean square error (MSE) based on the number of neurons in the hidden layer (NHN) during the phases of learning, validation and test. According to the values of MSE error learning, validation and test shown in Table I and Fig. 5, we can see that the minimum mean squared error is achieved when NHN $=4$. 


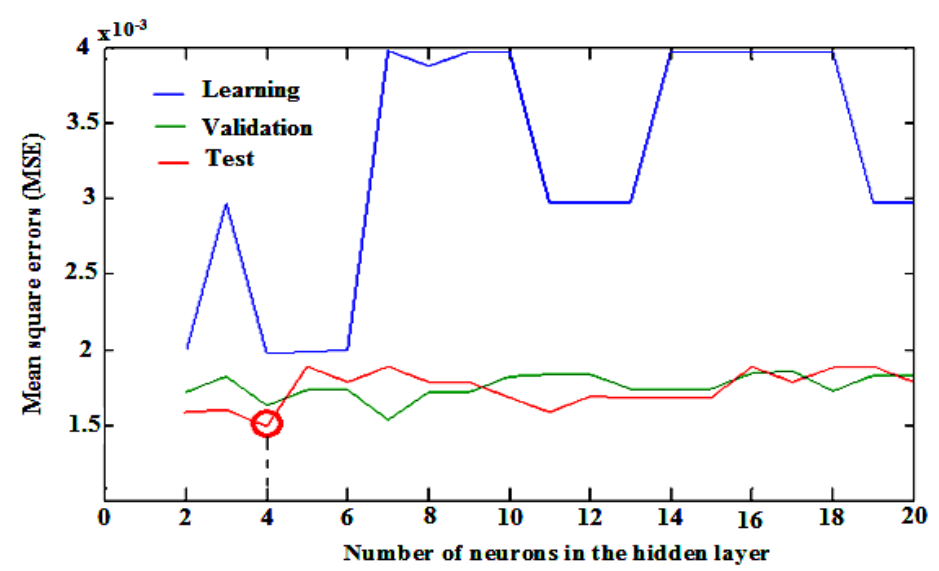

Fig. 5. Mean Square Error (MISE) depending on the number of neurons in the hidden layer relating to humidity

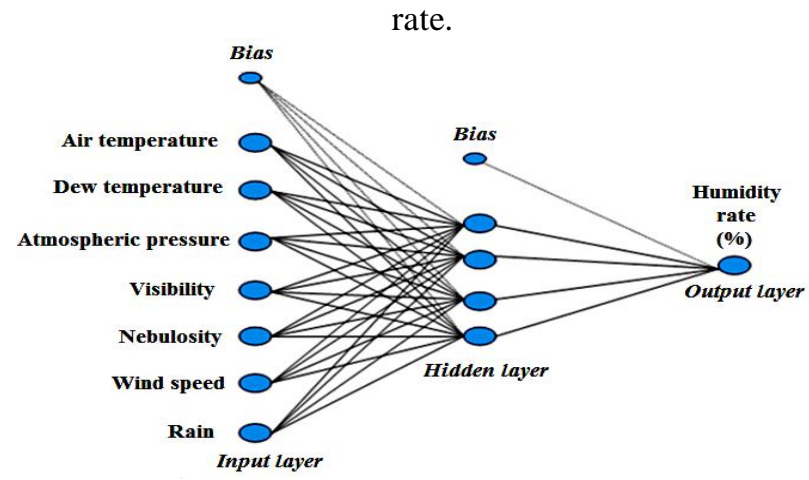

Fig. 6. Architecture of the neural network with three layers configuration [7-4-1] used in this study.

This shows that the network that consists of four neurons in the hidden layer is more efficient than a network consisting of 3 or $5,6, \ldots, 20$ neurons in the hidden layer for the prediction of moisture content (Fig.6).

\section{II.6 Modeling techniques}

The statistical analysis of our data base by the meteorological model of artificial neural networks was conducted by preliminary tests with several programs (Qnet, Pythia, Statistica, MATLAB ...). The results of this preliminary study showed that the MATLAB can be the most important simulation tool.

To interpret the results of the statistical model of neural network, a first application was performed on all data relating to 1248 days. In a second step, to justify the predictive quality of models, meteorological data are divided into three groups. The first group corresponds to $70 \%$ of the total data. This group will be used to drive the system. The second group corresponds to $15 \%$ of the total data; it will be used to validate the network and the remaining $15 \%$ that did not participate in the learning models will be used as an independent test of network generalization. It should be noted that these three groups of data were drawn from a random manner, among the total data. The results related to the correlation coefficients and mean square error, are given in Table II. These results show that the mean square error is quite small and similar for all three phases: learning, validation and test. They also show that the correlation is better for the three phases (close to unity).

Table II: Statistical parameters of ANN found with a configuration of the [7-4-1]

\begin{tabular}{|c|c|c|c|}
\hline Operation & Learning & Validation & Test \\
\hline Mean square errors (MSE) & 0.0014 & 0.0016 & 0.0016 \\
\hline correlation (R) & 0.98 & 0.97 & 0.97 \\
\hline
\end{tabular}




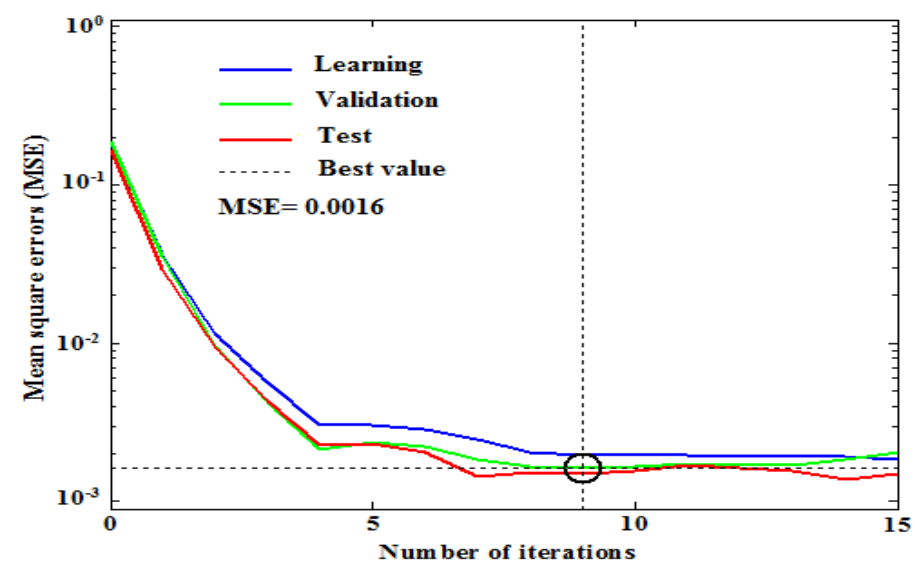

Fig.7. Evolution of the mean square error with four neurons in the hidden layer.

Fig.7 describes the workout of the network. After the ninth iteration, the desired result is achieved. With 4 hidden neurons, the three curves on the evolution of the mean square error of the three phases converge correctly to the minimum error. The network has been driven up to the stage of learning. This has been met after 15 iterations, it is interesting to continue learning until they reach this stage for testing in order to reduce the gradient and thus further improve our network (Fig. 8).
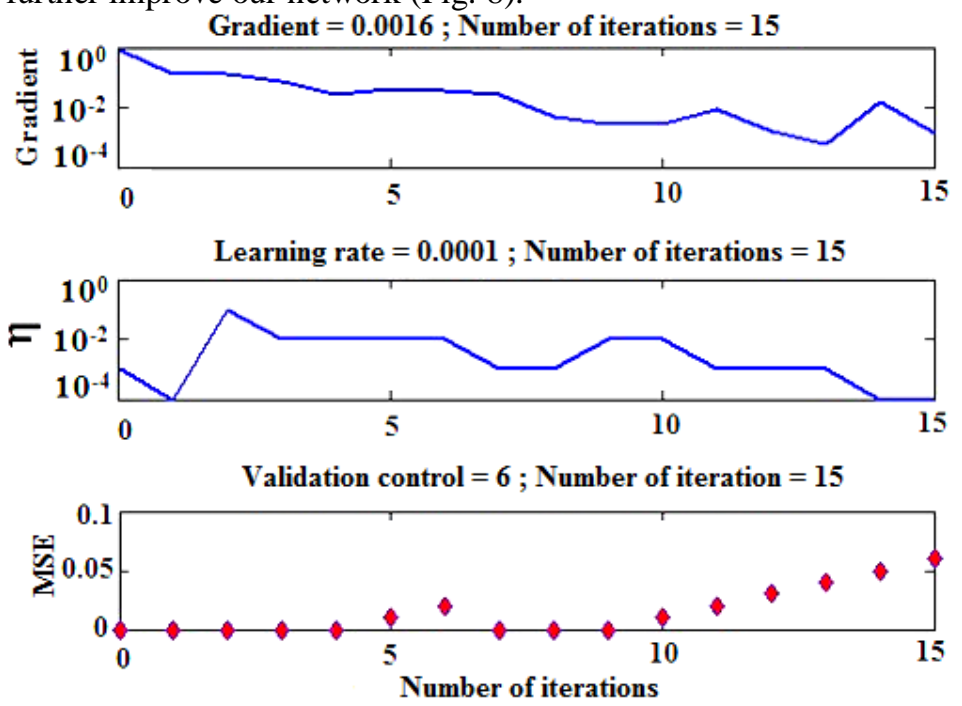

Fig. 8. Variations of the gradient of the error, the learning rate and the validation error depending on the number of iterations.

According to the results of fig. 8, we can infer the different values of learning parameters found in this study:

$\checkmark$ The learning parameters are as follows:

- Maximum number of iterations (Epochs) $=15$

- Root Mean Square Error (MSE) $=0.1$

- Rate of learning $(\eta)=0.0001$

- Gradient minimum $=0.0016$

\section{Result And Discussion}

\section{III.1 Evolution of the quality of learning and testing}

The developed ANN model gave a correlation coefficient of 0.98 for the training, which is equivalent to a mean square error of $0.19 \%$. For the validation period, the correlation coefficient was 0.97 , with a mean square error of $0.16 \%$.

For the test period, the correlation coefficient was 0.97 and the mean square error was $0.14 \%$, the correlation coefficients obtained by testing the validity of the model are relatively close to those obtained during the learning model (Fig. 9). 

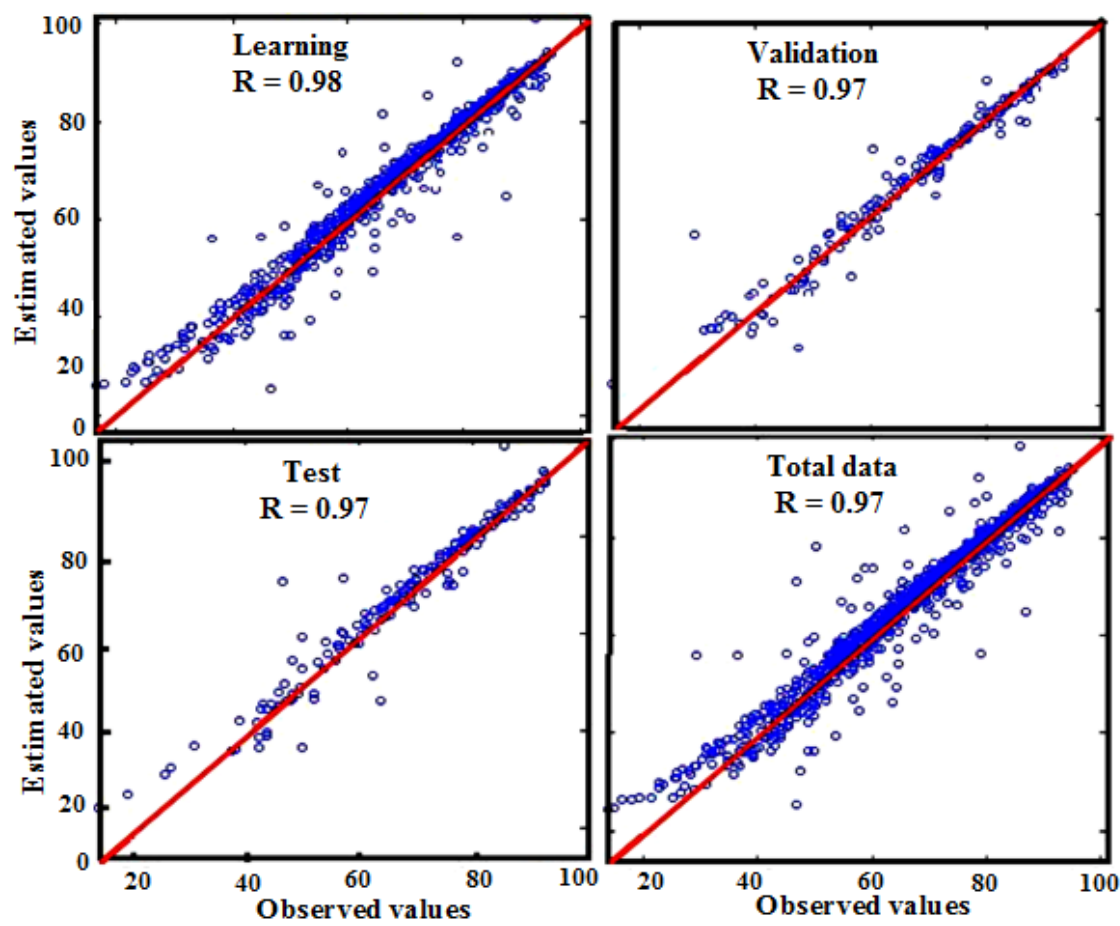

Fig.9. Relationship between observed moisture rates and those estimated by the models of artificial neural networks.

With neural networks, models have correlation coefficients almost equal to the unit $(\mathrm{R} \sim 1)$. This shows that there is a rapprochement between the measured values and those simulated by the neural model developed in this study. This model can be considered a highly effective tool in the study of weather prediction.

\section{III.2 Comparison between the two models (ANN and MLR)}

To compare the results between the two numerical methods (Neural and multiple linear regression), two performance indicators were calculated for each method: The correlation coefficient (R) and mean square error (MSE).

Correlation coefficients relating to learning, validation and test models established by multiple linear regression are significantly different from those for the model ANN (see Fig.10 and Table III). On the other hand, correlation coefficients calculated by the ANN were significantly higher (greater than 0.97), with the coefficients calculated against the MLR are lower (between 0.71 and 0.74 ).

This suggests that the meteorological parameters studied are related to the rate of humidity by nonlinear relationships.

Table III: Correlation coefficients obtained between observed moisture and those estimated by multiple linear regression and artificial neural networks.

\begin{tabular}{|c|c|c|c|c|c|c|}
\hline \multirow{2}{*}{ Methods } & \multicolumn{2}{|c|}{ Learning } & \multicolumn{2}{c|}{ Validation } & \multicolumn{2}{c|}{ Test } \\
\cline { 2 - 7 } & MSE & $\mathbf{R}$ & MSE & $\mathbf{R}$ & MSE & R \\
\hline MLR & 0.0510 & 0.71 & 0.0360 & 0.74 & 0.0400 & 0.73 \\
\hline ANN & 0.0019 & 0.98 & 0.0016 & 0.97 & 0.0016 & 0.97 \\
\hline
\end{tabular}




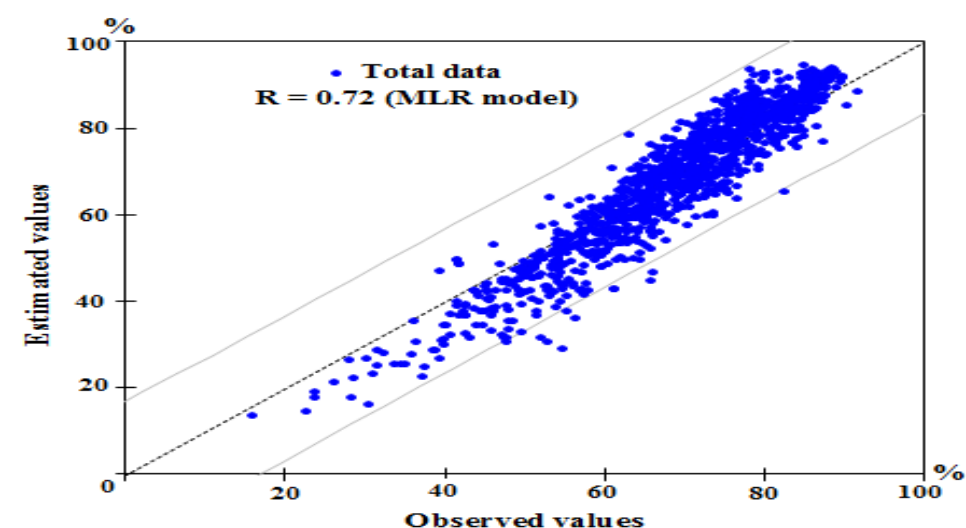

Fig.10. Relation between observed moisture rate values and values predicted by the linear model of multiple linear regression.

The results of this study show that artificial neural networks seem to give a slightly better adjustment to the data than that offered by the multiple linear regression.

\section{III.3 Studies of residuals}

The residue is the error committed by the models established by each individual method on a sample of model construction [3]. Fig.11 shows the residuals based on the simulated values during the learning phase, validation and testing. It shows that the residuals generally vary between $-5 \%$ and $5 \%$ for the ANN model. These results are very satisfactory and justify the use of an approach based on artificial neural networks in the prediction of moisture per day.

Residuals related to conventional models of multiple linear regression generally vary from $-10 \%$ to $10 \%$ with some residues exceeding these values.

This is in accordance with the results of some more recent studies that have shown that ANN models are performing compared to those established by the MLR models [5], [6] and [7].

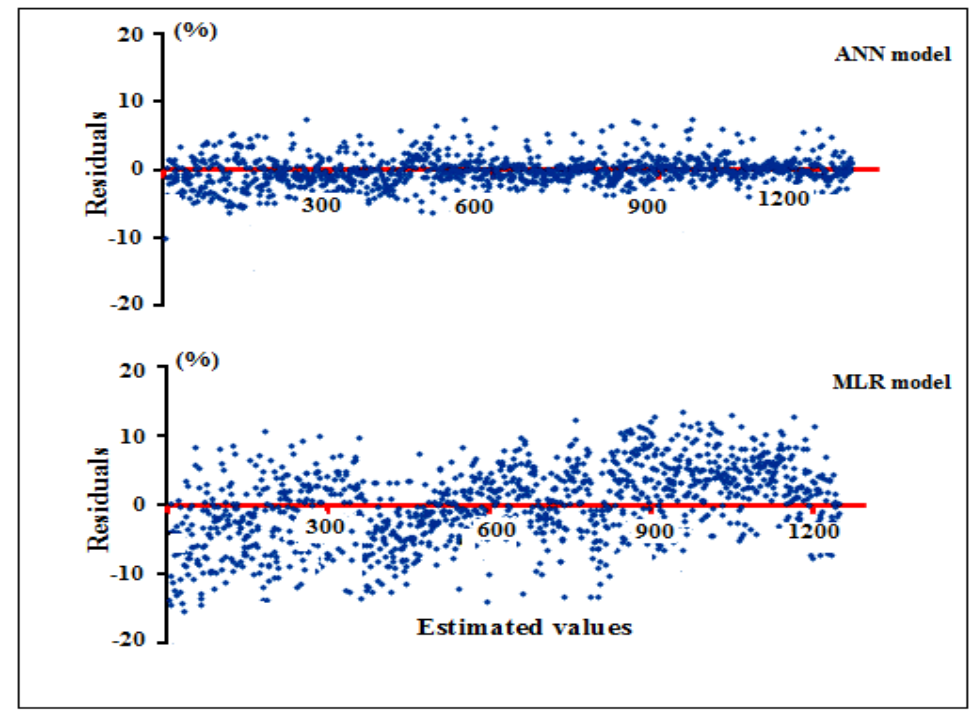

Fig.11. Distribution of residuals relating to MLR and ANN models for all data.

\section{Conclusion}

In this work we have presented a study about the use of artificial neural networks MLP type with a non-recurring for the prediction of moisture in the area of Chefchaouen, Morocco, from meteorological data. This study showed that the predictive models established by the ANN method are more efficient compared to those established by the method based on MLR. This shows that the meteorological parameters studied are related to the rate of humidity by nonlinear relations.

The results of this study open new perspectives to consider the development of other aspects of this project: making a prediction of the moisture close to two days including other meteorological parameters in the model of neural networks and work on other types of neuron networks: recurrent networks (radial function network (RBF) networks to competition ...). 


\section{REFERENCES}

[1] N. Cheggaga, F. Youcef Ettoumi, Estimation du potentiel éolien, Revue des Energies Renouvelables, SMEE'10 Bou Ismail Tipaza, 2010, $99-105$.

[2] M.Nohair, A. St-Hilaire et T. B. Ouarda, Utilisation de réseaux de neurones et de la régularisation bayésienne en modélisation de la température de l'eau en rivière. Revue des sciences de l'eau. Journal of Water Science, vol. 21, n 3, 2008, p. 373-382.

[3] C. Voyant, Prédiction de séries temporelles de rayonnement solaire global et de production d'énergie photovoltaïque à partir de réseaux de neurones artificiels, thèse pour l'obtention du grade de docteur en physique université de Corse-Pascal Paoli Ecole Doctorale Environnement et Société / UMR CNRS 6134 (SPE), 2011, 257p..

[4] Z. Ryad, R. Daniel et N. Zerhouni, Réseaux de neurones récurrents à fonctions de base radiales, RRFR, Revue d'Intelligence Artificielle, Volume X-n ${ }^{\circ} \mathrm{X} / 2002,2002$, p 1- 32 .

[5] P. Perez, A. Trier, Prediction of $\mathrm{NO}$ and $\mathrm{NO}_{2}$ concentrations near a street with heavy traffic in Santiago, Atmospheric Environment, 2001, 1783-1789.

[6] M. Bélanger, N. El-Jabi, D. Caissie, F. Ashkar et J.M. Ribi, Estimation de la température de l'eau en rivière en utilisant les réseaux de neurones et la régression linéaire multiple, Revue des sciences de l'eau, Rev.sci.Eau 18/3, 2005, 403-421.

[7] A. Abdallaoui, H. El Badaoui, Prédiction des teneurs en métaux lourds des sédiments à partir de leurs caractéristiques physicochimiques, Journal Physical and Chemical News, 2011, 90-97.

[8] L. Vecci, F. Piazza and A. Uncini, Learning and approximation capabilities of adaptive spline activation function neural networks. Neural Networks, 1998, 259-270.

[9] E. El Tabacha, L. Lancelot, I. Shahrour and Y. Najjar, Use of artificial neural network simulation metamodelling to assess groundwater contamination in a road project, Journal Elsevier Mathematical and Computer Modelling, 2007, 766-776.

[10] J. Rude, Développement d'un modèle statistique neuronal pour la description fine de la pollution par le dioxyde d'azote, Thèse d'Etat, 2008, 140p.

[11] V. Andronova, Utilisation de données météo et des réseaux de neurones pour la prédiction de vitesses de vent, 2006, 75p.

[12] A. Zouidi, A.Chaari, M. Stambouli and F. Fnaiech, Nonlinear continuous time modeling of a high pressure mercury vapor discharge lamp using feed forward back-propagation neural network, Yasmine Hammamet, Tunisie, IEEE 2004.

[13] Dreyfus, G., J. Marti nez, M. Samuelides, M. Gordon, F. Badran, S.Thiria et L. Hérault, Réseaux de neurones - Méthodologie et applications. Édition Eyrolles, Paris, France. 2002. 